\title{
Quality of Care in a Paediatric Intensive Care Unit of a Tertiary Care Teaching Hospital in New Delhi
}

\author{
Siddharth V' ${ }^{1}$, Gupta SK ${ }^{2}$, Lodha R $^{3}$, Satpathy $S^{4}$
}

\begin{abstract}
Introduction: This research aimed to study the outcome of patient care being rendered in Paediatric Intensive Care Unit (PICU) of an apex public sector teaching hospital of North India. Material and methods: A descriptive and observational study was carried out in PICU. Medical records of all admitted patients from January to June 2011 were analysed. Demographic, morbidity and mortality parameters were studied. PIM II score was calculated to assess the severity of illness. Results: In PICU, from January to June 2011, 110 patients were admitted. Almost equal number of patients got admitted in PICU through emergency ward/casualty and other inpatient areas. Of the total patients, 66\% (62) were male. Mean age of the patients admitted to PICU was 4.56 years. Three fourth patients admitted in PICU required mechanical ventilation and 652 days of mechanical ventilation was given. Patient's required mechanical ventilation on an average of 7.01 days. Almost one third, [36.61\% (21)] patients were reintubated. Majority $(78.7 \%)$ of the patients required oxygen support, while $72.3 \%$ (68) required vasopressor support. Prevalence of bed sore rate was $2.1 \%$. Only $2.1 \%$ patients required readmission within 72 hours. Mean hospital and PICU length of stay was 16.82 and 8.7 days respectively. Mean PIM 2 score of patients was $14.13 \%$ (range $0.2 \%$ to $86.9 \%$ ). Conclusion: $43.6 \%$ of patients died in PICU, while hospital mortality in PICU admitted patients was $47.2 \%$. Sepsis with septic shock was the major cause of mortality followed by pulmonary haemorrhage, disseminated intravascular coagulation. Standardised mortality was calculated to be 3.09 .
\end{abstract}

Key words: Mortality, Morbidity, Outcome of care, Paediatric Medicine Intensive Care Unit, Quality of care

\section{Introduction}

$\mathrm{T}$ he goal of paediatric intensive care is the surveillance and support of vital system functions in critically ill or injured children, and their eventual restoration to health ${ }^{1}$. During the past decades, the science of paediatric intensive care has progressed significantly. Paediatric intensive care units (PICUs) are regarded as making a substantial contribution to the health of children in developed and
${ }^{1}$ Dr. Vijaydeep Siddharth, Assistant Professor, Department of Hospital Administration, ${ }^{2} \mathrm{Dr}$. Shakti Kumar Gupta, Medical Superintendent, Dr. RP Centre for Ophthalmic Sciences, ${ }^{3} \mathrm{Dr}$. Rakesh Lodha, Professor, Department of Paediatrics, ${ }^{4}$ Dr. Sidhartha Satpathy, Professor, Department of Hospital Administration. All from the All India Institute of Medical Sciences, New Delhi, India.

\section{Address for correspondence}

Dr. Vijaydeep Siddharth, Assistant Professor, Department of Hospital Administration, AlIMS, New Delhi, India

Tel No; +919013844255

E-mail: dr.siddharthmamc@gmail.com

\section{Acknowledgements: None}

Funding: Nil

Conflict of Interest: This study was conducted as part of thesis submitted to All India Institute of Medical Sciences, New Delhi as partial fulfilment towards the award of degree in Masters of Hospital Administration. All authors fulfil the authorship criteria as laid down by ICMJE

Permission from IRB: Yes

\section{How to cite}

Siddharth V, Gupta SK, Lodha R, Satpathy S. Quality of Care in a Paediatric Intensive Care Unit of a Tertiary Care Teaching Hospital in New Delhi. J Nepal Paediatr Soc 2017;37(2):168173.

\section{doi: http://dx.doi.org/10.3126/jnps.v37i2.18133}

This work is licensed under a Creative Commons Attribution 3.0 License. 
developing countries ${ }^{2}$. Moreover, the availability of paediatric intensive care is regarded as a reflection of the quality of a country's paediatric medical care ${ }^{3}$. It is also important to understand that adults and children intensive care cannot be combined together because children's are not small people but different people and potential of psychological trauma to a child in a intensive care unit managing both adults and children ${ }^{4}$. Lately, the potential of paediatric intensive care has been enhanced by several factors i.e. developments in paediatric anaesthesia, medicine and surgery; technological and therapeutic advances in both neonatal and adult intensive care medicine; and improved understanding of paediatric physiology and mechanisms of severe illness in children ${ }^{5}$.

Measures of the effectiveness of paediatric intensive care should include physical and psychological sequelae, as well as the quality of life in survivors and their families ${ }^{6,7}$. It is important that every ICU formulates its indicators of quality as well as its standards to assess performance. The use of such indicators may help to identify problems and to develop methods to improve performance. Continuous measurement of the indicators is one method to assess an improvement of performance over time ${ }^{8}$. The end point in the quality improvement process is to describe the results, in terms of the primary outcomes. There are many important outcomes in critical care including mortality, length of stay etc $^{9}$. Predominantly studied outcome measures include mortality, morbidity, resource use and patient cantered outcomes (which incorporate patient preferences for their care and for their own functional status). A full evaluation of long-term outcomes is fundamental to the evaluation of the effectiveness of intensive care, in resource allocation, and in planning the long-term care of patients after discharge from the intensive care unit $(\mathrm{ICU})^{7,10}$.

In India there are very few studies on outcome of care, hence, this research aimed to observe the outcome of care being provided in paediatric medicine intensive care unit (PICU) of a large public sector tertiary care teaching hospital in Northern part of the India. This will help us in identifying the quality of care being rendered and measure which can help improve the same.

\section{Material and Methods}

This observational and descriptive study was carried out from October 2011 to July 2012 in PICU of a large public sector tertiary care teaching hospital in Northern part of the India after obtaining clearance from the Institute ethics committee. Retrospective analysis of medical records of all the admitted PICU patients was conducted from January 2011 to June 2011, to study the outcome of intensive care provided. The following outcome parameters were calculated. (Table 1)

Table 1: Outcome parameters for PICU

\begin{tabular}{l}
\hline Demographic and general profile of patients \\
\hline Morbidity \\
\hline - Length of stay \\
\hline ICU \\
\hline Hospital \\
\hline - Diagnosis \\
\hline - Post-operative/intervention complication \\
- Vasopressor support \\
- Oxygen administration \\
- Ventilator days \\
\hline - Unplanned reintubation within 48 hours \\
\hline - ICU readmission within $72 \mathrm{hours}$ \\
\hline Mortality \\
\hline - Hospital \\
\hline ICU \\
\hline - Standardised Mortality Rate (SMR) \\
\hline
\end{tabular}

Severity assessment of the admitted patients was done in PICU using PIM2 (paediatric index of mortality 2) score. Parameters for calculation of PIM2 was recorded from the information available in the medical records of the patients within one hour of admission and was calculated through open source online calculator. Data collected was analysed using Microsoft excel 2010.

\section{Results}

PICU is located within the paediatric medicine ward and can accommodate eight patients (five beds and three bassinets). It is pertinent to mention that there are separate Neonatal Intensive Care Unit (NICU) and Neonatal Surgical Intensive Care Unit (NSICU) within the Institute. It is a closed type ICU headed by Faculty, Department of Paediatric Medicine, who also has other assigned responsibilities of the department. In PICU, over a period of six months (January 2011 to June 2011), 110 patients were admitted, out of which medical records of only $97(88.18 \%)$ patients were available for study. PICU received majority of patients from Delhi $(46.81 \%)$ and remaining from the neighbouring states of Delhi i.e. Uttar Pradesh, Haryana etc. Majority of the patients who received PICU care presented on their own, while only few $(28.7 \%)$ were referred. Most of the patients who received PICU care were admitted in hospital from casualty $(85.1 \%)$, while only $13.8 \%$ were admitted through outpatient department. On analysis, it was found that almost equal number of cases got admitted in PICU through ward (51\%) and casualty $(49 \%)$. Only, three $(3.2 \%)$ patients were medico legal cases. 
Of the total patients admitted in PICU, 66\% (62) were male, rest were females i.e. $34 \%$ (32). The mean and median age of the patents admitted to PICU was 4.56 and 2.05 years (range 0-17years) respectively. Mean and median age of female patients was lower than that of males. Referred patients and patient's resident of states other than Delhi were younger in age. (Table 2) In all the age groups proportion of males admitted to PICU was higher than the females. On analysis of birth history, it was found that $78 \%$ patients were delivered in healthcare and only $9.21 \%$ were preterm.

A total of 652 days of mechanical ventilation was provided to $75.53 \%$ (71) patients requiring mechanical ventilation during their stay. Mean (median) days of mechanical ventilation of patients admitted in PICU were 7.01 (3) days (range 1 -66 days). Patients of age group of 1 to less than 5 years, patients from states other than Delhi and patients who succumbed to the illness required longer duration of mechanical ventilation. Of the total patients requiring mechanical ventilation, $36.61 \%(21)$ patients were reintubated within 48 hours of extubation. Of the total admitted patients $78.7 \%$ patients required oxygen support during their PICU stay and $72.3 \%$ (68) required vasopressor support. Prevalence of bed sore rate in PICU was $2.1 \%$. Only two patients $(2.1 \%)$ were readmitted within 72 hours during their hospital stay after getting transferred out from PICU. On analysis, it was found that prevalence of morbidity indicators was comparatively more common in males than females except for the readmission and bed sore rate which were equal in both the sexes, however, statistical significance was not established. (Figure 2)

Severity of illness among patients admitted in PICU was assessed using paediatric index of mortality II score (PIM 2). Mean (median) PIM 2 score of patients admitted at PICU was $14.13 \%$ (5.45\%). Predicted mortality amongst patients admitted in PICU ranged from $0.2 \%$ to $86.9 \%$. PIM2 score was highest among the patients of less than one year of age admitted in PICU. Mean PIM 2 score was more in males (15.83\%) while median PIM 2 score (5.45\%) was same across the gender. Mean PIM 2 score in non-referred patients was higher than referred patients and overall PIM 2 score of admitted patients. (Table 3)

Mean (median) hospital length of stay in patients who received PICU care was 16.82 (13) days (range 1 -84 days) respectively. Mean (median) PICU, length of stay was calculated to be 8.7 (4) days (range 1 -71 days) respectively. Length of stay was longer in males compared to females, referred compared to nonreferred patients, and patients who were transferred out from PICU compared to those who died. Bed occupancy rate in paediatric intensive care unit was calculated to be $72.39 \%$.

Of the total admitted patients, $43.6 \%$ (41) expired, while rest of the patients were transferred out to other inpatient care areas for further management. More than half $(53.2 \%)$ of the patients were transferred out from PICU to paediatric medicine ward. Of the total patients $(56.4 \%)$ transferred out, $3.6 \%$ (3) died during their hospital stay before they could be finally discharged from the hospital. Approximately half of the patients $(47.2 \%)$ admitted in PICU died during the hospital stay. Mortality pattern did not vary with either gender or referral status of the patient. Proportionately higher mortality was observed in patients of less than 1 year of age and patients from neighbouring states. Sepsis along with septic shock was found to be the major cause of mortality in PICU patients followed by pulmonary haemorrhage, disseminated intravascular coagulation. Standardised mortality was calculated to be 3.09 .

Table 2: Morbidity amongst patients admitted in PICU

\begin{tabular}{|c|c|c|c|c|c|c|c|c|c|c|}
\hline \multirow{2}{*}{ Variable } & & \multirow[b]{2}{*}{$\mathbf{N}$} & \multicolumn{2}{|c|}{ Age } & \multicolumn{2}{|c|}{ Hospital LOS } & \multicolumn{2}{|c|}{ PICU LOS } & \multicolumn{2}{|c|}{ Ventilator days } \\
\hline & & & M & $\mathbf{m}$ & $\mathbf{M}$ & m & M & $\mathrm{m}$ & M & m \\
\hline \multirow{4}{*}{ Age Group } & & & & & & & & & & \\
\hline & 0 to $<1$ year & 37 & & & 14.5 & 9.0 & 13.0 & 7.0 & 7.7 & 4 \\
\hline & 1 to $<5$ years & 17 & & & 21.8 & 15.0 & 13.0 & 7.0 & 10.8 & 5 \\
\hline & $>5$ years & 40 & & & 16.9 & 13.5 & 6.0 & 2.0 & 4.7 & 1 \\
\hline \multirow{2}{*}{ Gender } & Male & 62 & 4.8 & 4.0 & 19.0 & 14.0 & 9.7 & 5.5 & 7.9 & 4 \\
\hline & Female & 32 & 4.0 & 1.2 & 12.7 & 8.5 & 6.8 & 4.0 & 5.3 & 1 \\
\hline \multirow{2}{*}{ Referral status } & Referred & 27 & 2.7 & 0.5 & 18.6 & 16.0 & 10.6 & 6.0 & 7.8 & 3 \\
\hline & Non referred & 67 & 5.3 & 4.6 & 16.1 & 13.0 & 8.0 & 4.0 & 6.7 & 3 \\
\hline \multirow{2}{*}{$\begin{array}{l}\text { Status at discharge } \\
\text { from ICU }\end{array}$} & $\begin{array}{l}\text { Transferred to paediatric } \\
\text { medicine wards }\end{array}$ & 50 & 5.1 & 4.0 & 20.8 & 15.0 & 9.8 & 4.0 & 6.9 & 1 \\
\hline & Expired & 41 & 3.5 & 0.5 & 11.5 & 7.0 & 7.9 & 5.0 & 7.4 & 4 \\
\hline
\end{tabular}

$\mathrm{M}=$ Mean $\mathrm{m}=$ Median 
Table 3: Expected and observed mortality among PICU patients

\begin{tabular}{|c|c|c|c|c|c|c|}
\hline \multirow{3}{*}{ Parameter } & \multirow{3}{*}{ Variable } & \multirow{2}{*}{$\mathbf{N}$} & \multicolumn{2}{|c|}{ Predicted Mortality * (\%) } & \multirow{2}{*}{$\mathbf{N}$} & \multirow{2}{*}{$\begin{array}{c}\text { Observed } \\
\text { Mortality (\%) }\end{array}$} \\
\hline & & & Mean & Median & & \\
\hline & & 94 & 14.13 & 5.45 & 41 & 43.60 \\
\hline \multirow{3}{*}{ Age Group } & 0 to $<1$ year & 37 & 15.23 & 7.5 & 23 & 62.16 \\
\hline & 1 to $<5$ years & 17 & 9.29 & 4.7 & 4 & 23.53 \\
\hline & $>5$ years & 40 & 15.16 & 5 & 14 & 35.00 \\
\hline \multirow{2}{*}{ Gender } & Male & 62 & 15.83 & 5.45 & 27 & 43.54 \\
\hline & Female & 32 & 10.84 & 5.45 & 14 & 43.75 \\
\hline \multirow{2}{*}{ Referral status } & Referred & 27 & 10.39 & 5 & 12 & 44.44 \\
\hline & Non referred & 67 & 15.63 & 6.2 & 29 & 43.28 \\
\hline \multirow{2}{*}{$\begin{array}{l}\text { Status at } \\
\text { discharge from } \\
\text { ICU }\end{array}$} & $\begin{array}{l}\text { Transferred to paediatric } \\
\text { medicine wards }\end{array}$ & 50 & 9.75 & 4.45 & & \\
\hline & Expired & 41 & 20.11 & 9.6 & & \\
\hline
\end{tabular}

*Predicted mortality was calculated using Paediatric Index of Mortality II (PIM II) Observed

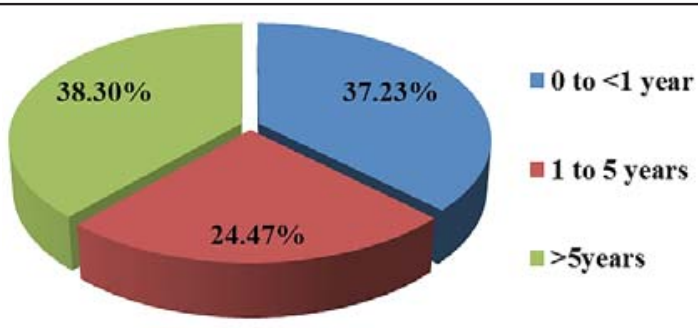

Fig 1: Age group of patients admitted to PICU

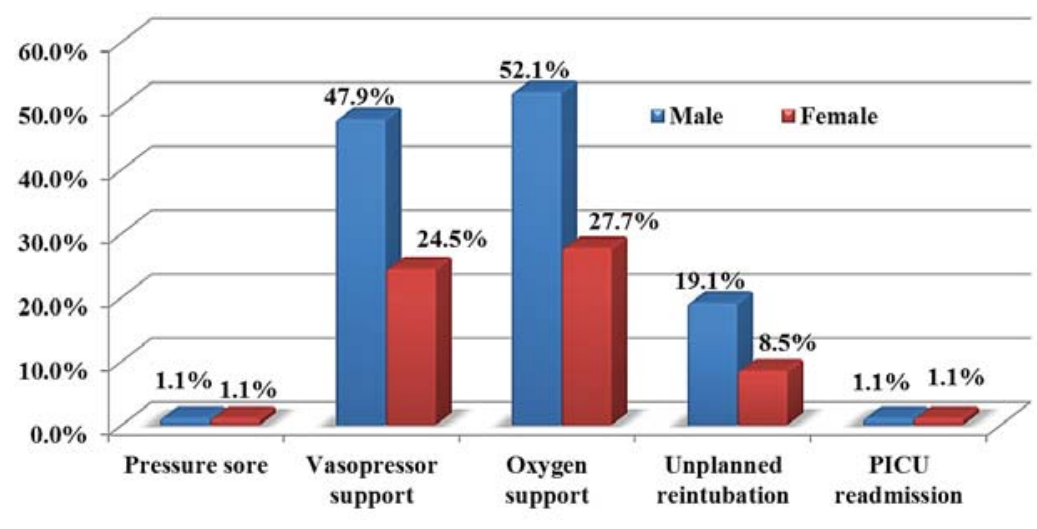

Fig 2: Comparison of morbidity indicators among male and females

\section{Discussion}

This research aimed to observe the outcome of care in a critical care setting, which may reflect upon the quality of care being rendered and the scope of improvement in same. A total of $75.53 \%$ (71) patients required mechanical ventilation during their PICU stay. Similarly, in a report published in year 2010 by University of Leeds and Leicester, it was reported that $83 \%$ of PICU admissions received invasive ventilation ${ }^{11}$. However, in another report of PICANet, Scotland it was reported that only $54 \%$ of PICU admissions received invasive ventilation ${ }^{12}$. While in contrast, a study done in Brazil, of
964 patients admitted during the five-year period, only $283(29.3 \%)$ required invasive mechanical ventilation ${ }^{13}$. A study done in Haiti, reported that $55 \%$ (11) patients received mechanical ventilation with a median duration of 2 days (interquartile range, 1-4 days) ${ }^{14}$. Almost one third $(36.61 \%)$ patients, were reintubated within 48 hours of extubation. Unplanned tracheal extubation is an important quality issue in current medical practice, as it is a common occurrence in paediatric intensive care units. However, a study done in developing nations, PICU reported unplanned extubation events occurring in $18.7 \%$ (52) patients $^{13}$. 
In a study, it was observed that following implementation of the continuous quality improvement programme over a period of five years, the overall incidence of unplanned extubation decreased from 2.9 unplanned extubation per 100 intubated patient days to 0.6 ( $p=0.0001)$. Although mortality was similar to that of children who did not experience an unplanned extubation, those with an unplanned extubation had a significantly longer duration of mechanical ventilation, longer stay in the intensive care unit, and longer hospital stay ${ }^{13}$. It is much desired that quality initiatives needs to be undertaken to bring down the unplanned extubations, duration of mechanical ventilation, length of stay etc. which has a bearing on overall outcome of care.

In this study, mean (median) PICU length of stay was 8.7 (4) days (range 1 -71 days) respectively. While, a study done in Haiti, median PICU length of stay was only 2.5 days (interquartile range, 1-4 days) ${ }^{14}$. Median paediatric ICU length of stay in Australia and New Zealand in 2008 was reported to be 1.3 days $^{15}$. Mean (median) PIM 2 score of patients admitted at PICU was $14.13 \%$ (5.45\%) (range $0.2 \%$ to $86.94 \%$ ) and $43.6 \%$ (41) of total PICU patients succumbed to the illness during the course of their treatment, while hospital mortality among PICU admitted patients was observed to be $47.2 \%$. Significant difference was observed between predicted and observed mortality, which may be because of the under predicted mortality by PIM 2, which could be because of the differences in the patient profile and greater load of severity of illness being managed with lesser resources-both physical and human-and differences in the quality of care ${ }^{16}$. Sepsis along with septic shock was found to be the major cause of death followed by pulmonary haemorrhage, disseminated intravascular coagulation in patients who died in PICU.

While contrastingly, very low predicted risk of death (3.6\%) by PIM2 for paediatric patients and very low crude mortality (range $2.9 \%-5.7 \%$ ) has been reported in developed countries like Australia, New Zealand, United Kingdom ${ }^{11,12,15}$.

\section{References}

1. Downes JJ. The historical evolution, current status, and prospective development of pediatric critical care. Crit Care Clin 1992;8(1):1-22.

2. Köroğlu TF, Atasever S, Duman M. A survey of pediatric intensive care services in Turkey. Turk $J$ Pediatr 2008;50(1):12-7.
Organisation of PICU servicess neeeds stregthening despite adequate machinery and equipment availability, in order to improve upon the quality of care being rendered in PICU to bring at par with that of the developed countries. The structural aspects of paediatric intenisve care unit have also played a role in limiting the organisation of service delivery as per the standard guidelines. The system of policies and procedures needs to be strengthened.

It is also pertinent to mention that the PICU under study, was not a part of the building when this healthcare institution was planned but was later on retrofitted as the institute grew, to meet the patient care requirements. Hence, the manner in which this PICU has been designed and organised is not an ideal setting and may be falling short of standard guidelines. However, this PICU is adequately equipped when it comes to machinery and equipment but lacks in human resource prscribed by National and International guidelines on the subject. These design and organisational factors has a bearing on the delivery and outcome of care as well, and can be responsible for longer length of stay, mechnical ventilation, and may be mortality as well. This huge difference in mortality among patients admitted in PICU in this study located in lower middle income country viz a viz developed country can be attributed to the manner in which healthcare systems have been organised, availability, affordability and accessibility of the healthcare delivery system (healthcare facilities, human resource for health, equipment, medicines, budget available etc.), emergency medical service systems etc ${ }^{14}$.

\section{Conclusion}

The morbidity and mortality measures observed in this study are not very encouraging but identifies some important issues which needs to be addresssed. The fact that being an apex public sector healthcare institution in a developing country, it is overwhelmed by the sheer volume of critically ill cases being recieved. Further, the deficiencies in the overall healthcare delivery system of the country acts as hindrance in delivering quality healthcare.

3. López-Herce J, Sancho L, Martinón JM. Study of paediatric intensive care units in Spain. Spanish Society of Paediatric Intensive Care. Intensive Care Med 2000;26(1):62-8.

4. Ramesh S. Paediatric intensive care-update. Indian J Anaesth 2003;47:338-44.5.

5. Bennett NR. Paediatric intensive care. $\mathrm{Br} J$ Anaesth [Internet]. 1999;83(1):139-56. 
6. Morrison AL, Gillis J, O'connell AJ, Schell DN, Dossetor DR, Mellis C. Quality of life of survivors of pediatric intensive care. Pediatr Crit Care Med 2002;3(1):1-5.

7. Knoester H, Grootenhuis MA, Bos AP. Outcome of paediatric intensive care survivors. Eur $J$ Pediatr 2007;166(11):1119-28.

8. Thijs LG. Continuous quality improvement in the ICU: general guidelines. Intensive Care Med 1997;23(1):125-7.

9. Barbieri C, Carson SS, Amaral AC. Year in review 2007: Critical Care--intensive care unit management. Crit Care 2008;12(5):229.

10. Taylor A, Butt W, Ciardulli M. The functional outcome and quality of life of children after admission to an intensive care unit. Intensive Care Med 2003;29(5):795800.

11. Draper E, Mckinney P, Lamming C, McShane P, Parslow R, Shearing A. Welsh Report of the Paediatric Intensive Care Audit Network January 2007December 2009. Leeds; 2010
12. Draper E, Mckinney P. National Report for Scotland of the Paediatric Intensive Care Audit Network January 2007- December 2009. Leeds; 2010.

13. da Silva PSL, de Aguiar VE, Neto HM, de Carvalho WB. Unplanned extubation in a paediatric intensive care unit: impact of a quality improvement programme. Anaesthesia 2008;63(11):1209-16.

14. Brogan T V, Hertzig J, Kim K, Wurm G, Roberts J, Fink EL. Intensive care for infants and children in Haiti in April 2010. Paediatr Crit Care Med 2011;12(4):393-7.

15. First Annual Report of the Australian and New Zealand Intensive Care Society (ANZICS) Centre for Outcome and Resource Evaluation (CORE). Carlton South VIC; 2008.

16. Thukral A, Lodha R, Irshad M, Arora NK. Performance of Pediatric Risk of Mortality (PRISM), Pediatric Index of Mortality (PIM), and PIM2 in a pediatric intensive care unit in a developing country. Pediatr Crit Care Med 2006;7(4):356-61. 